\title{
BIOMATEMÁTICA: MÉTODOS E LIMITAÇÕES
}

José Roberto Castilho PIQUEIRA ${ }^{1}$

- RESUMO: Este trabalho trata de uma visão panorâmica dos principais métodos utilizados, atualmente, na modelagem matemática de processos biológicos. Esses métodos são muito variados, com enfoques que, às vezes, são determinísticos, utilizando a teoria dos sistemas dinâmicos e, às vezes, são probabilísticos, lançando mão das equações diferenciais estocásticas. Em todos eles, entretanto, três fatores inerentes aos processos biológicos não podem deixar de ser considerados: a complexidade, a imprevisibilidade e a diversidade de escalas temporais.

- PALAVRAS-CHAVE: Sistemas dinâmicos; complexidade; imprevisibilidade; equações diferenciais; mecânica clássica; mecânica quântica; termodinâmica.

\section{Introdução}

Nos últimos anos, as ciências biológicas têm recebido da matemática uma interessante contribuição que, aparentemente, originou-se da necessidade crescente de se processar, eficientemente, os dados clínicos e de laboratório, na tentativa de extrair de tais dados a máxima quantidade de informação possivel, criando processos computacionais cada vez mais potentes. ${ }^{2}$

Essas contribuições, não apenas trabalhando como ferramentas de natureza estatística, tão importantes nos testes de validação de hipóteses a respeito dos dados, constituem uma empreitada mais ousada de criação de modelos de sistemas que possam descrever, predizer e prescrever algum recorte dos processos biológicos.

Não se trata de encarar a matemática como ferramenta para fazer contas e interpretar gráficos, e sim utilizá-la nas modelagens efetivas dos processos, proporcionando conjuntos de equações diferenciais em problemas de dinâmica populacional, fisiologia e bioquímica, além de modelos estocásticos representativos de processos de nível celular e molecular. ${ }^{3}$

\footnotetext{
1 Departamento de Engenharia Eletrônica - EP-USP; IEA-USP - 05508-900 - São Paulo - SP.

2 Holden, 1993; Cvitanovic, 1989; Mittenthal \& Baskin, 1992; Blumenfeld, 1980; Ruele, 1991; Gleick, 1988.

3 Mittenthal \& Baskin, 1992; Engel, 1978; Bai-Lin, 1990; Kauffman, 1993.
} 
Tal empreitada tem redundado em sucesso e contribuído de maneira efetiva para o desenvolvimento da biologia teórica, conforme se observa na literatura científica da área (Murray, 1993).

É importante deixar claro que a biomatemática não pode ser confundida com a biofísica ou bioquímica, pois não procura explicar os processos biológicos por meio de sua decomposição sucessiva em processos físicos e químicos de níveis de organização gradativamente inferiores (Engel, 1978).

Os dados medidos devem ser os elementos essenciais da criação dos modelos, gerando equações que, para certas faixas de ocorrência dos processos, sejam compativelmente ajustáveis.

Entretanto, deve-se observar que, atualmente, o processo está sendo revertido. Não é só a biologia que se beneficia dos métodos da matemática, mas sim esta que inicia um novo estágio de procura de problemas para o aprimoramento de suas teorias.

Acostumada a encontrar na física e na química suas situações problemáticas, a matemática tenta, agora, desenvolver metodologia para contemplar de maneira satisfatória as duas principais questões relativas ao processo biológico: a complexidade e a irreversibilidade.

É desse movimento que este artigo trata, iniciando com algumas idéias a respeito do uso da teoria dos sistemas dinâmicos em problemas de biologia, enfatizando suas limitações e apresentando algumas alternativas teóricas de solução de tais limitações.

Nessas situações, os modelos dinâmicos que geram comportamentos caóticos tornam-se extremamente convidativos, proporcionando uma eventual explicação para a questão da complexidade.

A o falar de complexidade, parece impossivel não distinguir a complexidade funcional da estrutural. Isto será, ao menos tangencialmente, abordado neste artigo.

Os modelos probabilísticos e as equações diferenciais estocásticas, largamente utilizados na modelagem de processos de difusão, serão também tratados e sua utilidade discutida.

Por se tratar de um simples resumo, algumas questões não serão abordadas. A principal delas é a discussão do tratamento dos dados biológicos, bem como a descrição dos diversos métodos para sua coleta.

\section{Noções sobre sistemas dinâmicos}

O estabelecimento de equações para modelar processos naturais é busca antiga do homem, e talvez tenha começado a ter sucesso na proposição das Leis de Newton da mecânica. Basta conhecer o estado inicial de um sistema e todas as forças que nele atuam para que todo o comportamento futuro esteja univocamente determinado pela resolução das equações provenientes de tais leis (Fiedler-Ferrara \& Prado, 1994; Hirsh \& Smale, 1974). 
Essa natureza determinística de tal metodologia, aplicada com sucesso a uma imensa quantidade de problemas reais, popularizou o sonho de matematização do universo entre os cientistas. O obsessivo desejo do homem de prever e manipular o futuro encontrava em tais teorias a esperança de realização.

Ao longo dos séculos XVIII e XIX, físicos e matemáticos aprimoraram o uso das Leis de Newton, desenvolvendo métodos eficientes para a resolução dos mais diversos tipos de equações diferenciais, originárias, fundamentalmente, da aplicação dos métodos de Newton aos problemas mecânicos. Entretanto, isso praticamente não atraiu os pesquisadores de outras áreas para o uso de tais metodologias. Duas coisas pareciam faltar para compatibilizar essas idéias com a natureza que, efetivamente, nos rodeia: o imponderável e o complexo.

Que espaço sobraria para o imponderável cotidiano das ciências da cultura ou para o complexo cotidiano das ciências biológicas? Como levar em consideração tais fatos nas equações? E, mais ainda, as Leis de Newton consideram situações totalmente reversiveis, não levando em conta as marcas que o tempo deixa em qualquer processo biológico ou cultural.

Como compatibilizar tais questões é algo que ocupa o dia a dia dos filósofos da ciência e dos pesquisadores de vanguarda das diversas áreas.

Aqui, nenhum aspecto cultural será discutido, tratando-se apenas de mostrar como algumas ferramentas próprias da matemática têm sido aplicadas com o intuito de contornar as dificuldades impostas pela complexidade e irreversibilidade biológica.

Nesse contexto, a teoria dos sistemas dinâmicos, versão evoluída dos métodos de resolução de equações diferenciais, desempenha papel fundamental que se origina na conjectura do início do século XX, devida a Poincaré, de que sistemas mecânicos descritos por equações de caráter determinístico podem apresentar comportamentos totalmente imprevisíveis e, aparentemente, aleatórios. ${ }^{4}$

Essa conjectura, correta para sistemas não lineares, com a ajuda dos métodos computacionais, produziu aquilo que se popularizou como teoria do caos, caso particular da teoria das bifurcações dos sistemas dinâmicos. A teoria de bifurcações trata os sistemas dinâmicos como famílias de equações diferenciais parametrizadas e observa que variações nos parâmetros produzem mudanças qualitativas nas soluções, sem que o formato original das equações seja alterado.

São esses pontos que têm atraído a biologia teórica em direção à matemática, em particular à teoria dos sistemas dinâmicos. As equações podem, de alguma forma, levar em conta os comportamentos imponderáveis e complexos, e questões como variabilidade, adaptação e ontogênese de processos talvez possam ser modeladas como influências de parâmetros na qualidade das soluções.

Aqui surge uma questão importante de uniformização de linguagem. Entendese por parâmetro de um processo ou modelo, uma variável cuja taxa de variação a

4 Holden, 1993; Cvitanovic, 1989; Bai-Lin, 1990; Fiedler-Ferrara \& Prado, 1994; Hirsh \& Smale, 1974; Ott, 1993. 
ser considerada é desprezivel. Por outro lado, as chamadas variáveis de estado são aquelas cuja taxa de variação não pode ser desprezada no processo em estudo. Assim, uma mesma variável de um dado processo biológico pode ser considerada um parâmetro ou uma variável de estado, dependendo da escala temporal adotada. Esse é outro aspecto que complica o uso das ferramentas habituais de matemática nos processos biológicos. A necessidade de se trabalhar com escalas temporais discrepantes no estudo de um mesmo processo ou modelo. Este artigo segue tentando abordar, de maneira conceitual, os problemas supra descritos, dividindo-os nos seguintes tópicos:

- Complexidade estrutural e funcional sob o ponto de vista da teoria dos sistemas dinâmicos.

- Variedade de escalas temporais sob o ponto de vista da teoria dos sistemas dinâmicos.

- Irreversibilidade e a necessidade das abordagens termodinâmicas.

- Imprevisibilidade de comportamentos: equações deteminísticas e estocásticas.

\section{Complexidade estrutural e funcional}

Complexidade é matéria que diz respeito a um grande número de ramos de pesquisa, como computação, fisiologia, neurologia, física, química, engenharia, ciências sociais e tantos outros, cuja citação excederia o número de páginas disponíveis para este trabalho. ${ }^{5}$

Recentemente, tornou-se até algo de bom-tom científico trabalhar como pesquisador das ciências da complexidade. Porém, alguma matemática é necessária para tornar as questões mais claras e manipuláveis.

Embora a diversidade de aplicações desses conceitos seja grande, algumas idéias relativamente precisas podem ser apresentadas, principalmente, distinguindo o que se denomina complexidade estrutural do que se denomina complexidade funcional.

Seja um fenômeno natural qualquer e seja um conjunto de variáveis que represente esse fenômeno em um determinado instante. Alguém pode contestar, dizendo que seu problema é tão complexo que não se pode, sequer, estabelecer o número de variáveis que o seu estudo requer.

Isso não é dificuldade. É possivel esperar por melhorias nas abordagens, até que essa classe de representação possa ser aplicada ao problema.

Assim, é possível ter um número finito $\mathrm{N}$ de variáveis de estado descrevendo um sistema objeto de estudo e, quanto mais se conhece o comportamento desse conjunto de variáveis, à medida que o tempo passa, mais se conhece sobre o sistema.

5 Mittenthal \& Baskin, 1992; Kauffman, 1993; Weber et al., 1988; Thompson, 1980; Thom, 1975; Solomon, 1981. 
Utilizando um pouco de formalismo, uma função vetorial do tempo $x(t)$ associa, a cada instante, um conjunto de $\mathrm{N}$ números reais, descrevendo o estado do sistema. A complexidade estrutural está associada a esse número N. Quanto maior ele for, mais complexa será a estrutura do sistema.

Entretanto, deve-se notar que complexidade estrutural não implica, necessariamente, comportamentos inesperados ou complicados. Caso, por exemplo, o sistema possa ser considerado linear, suas soluções não apresentarão nenhum aspecto inusitado (Hirsh \& Smale, 1974; Wiggins, 1990).

Há uma questão mais profunda a ser discutida relacionada com os valores que cada componente da função vetorial de estados pode assumir.

Para tanto, deve-se considerar que a cada componente $\mathrm{x}_{\mathrm{i}}$ da função vetorial de estados podem ser associados $\mathrm{N}_{\mathrm{i}}$ soluções de regime permanente diferentes. Isto é, deve-se raciocinar que o sistema biológico é descrito por $\mathrm{N}$ funções do tempo $\mathrm{x}_{1}, \mathrm{x}_{2}$, $\ldots \ldots, \mathrm{X}_{\mathrm{N}}$, cada uma delas assumindo, respectivamente, $\mathrm{N}_{1}, \mathrm{~N}_{2}, \ldots . . \mathrm{N}_{\mathrm{N}}$ soluções atratoras possiveis.

A complexidade funcional está associada ao produto dos números $N_{1}, N_{2}, \ldots$. $\mathrm{N}_{\mathrm{N}}$ uma vez que ele representa o número global de soluções atratoras para o sistema como um todo.

Combinando complexidade estrutural com complexidade funcional, têm-se a complexidade efetiva do sistema, do ponto de vista de seu comportamento dinâmico.

Essa é uma visão do problema que pode ser útil à biologia: o sistema biológico modelado por uma equação diferencial de ordem N, governada por parâmetros. Variações nesses parâmetros, embora não mudem a formulação das equações, introduzem mudanças qualitativas nas soluções atratoras, variando a complexidade do sistema.

\section{Diversidade de escalas temporais e irreversibilidade}

Conforme foi discutido no item anterior, a teoria dos sistemas dinâmicos não lineares proporciona uma boa ferramenta para o tratamento de alguns problemas biológicos.

A utilização de tal teoria tem sido bem-sucedida nos problemas de competição entre espécies, da dinâmica populacional, com o desenvolvimento de vários modelos sofisticados a partir das equações de Lotka-Volterra. ${ }^{6}$

Esses modelos adquiriram um grau de qualidade tão interessante que passaram a ser usados em todo tipo de questão que envolve competição entre populações, tais Esses modelos adquiriram um grau de qualidade tão interessante que passaram a ser usados em todo tipo de questão que envolve competição entre populações, tais como sensibilidade bacteriana em hospitais e controle do estabelecimento de sono REM e não-REM, a partir de disparos de neurônios de ativação e desativação (Kuramoto, 1980; Hobson, 1988).

6 Holden, 1993; Solomon, 1981; Bassanezi \& Ferreira Júnior, 1988. 
Além disso, em virtude de características periódicas e aperiódicas dos processos biológicos, vários modelos usando osciladores proporcionam níveis de predição surpreendente em problemas de fisiologia, tanto animal como vegetal (Mittenthal \& Baskin, 1992; Kuramoto, 1980).

Particularmente, essa família de modelos tem se apresentado tão eficiente que já dá resultados de natureza clínica, em previsões de epidemias, tratamento de doenças periódicas e previsões de patologias em indivíduos e populações. ${ }^{7}$

Apesar de tudo isso, todo o estudo realizado dessa maneira pressupõe processos dinâmicos rápidos, modelados por equações diferenciais ordinárias não lineares, considerando que algumas variáveis do processo assumem valores estáveis, passando a fazer o papel de parâmetros.

A visão cronobiológica do desenvolvimento e estabelecimento de ciclos a o longo da vida não faz parte desta abordagem, necessitando incluir equações diferenciais adicionais, que levem em conta dinâmicas lentas, relacionadas com variações de parâmetros ao longo do tempo.

Assim, parece que a teoria dos sistemas dinâmicos é ferramenta relativamente satisfatória para a explicação de fenômenos biológicos, uma vez que pode lançar mão de equações diferenciais com escalas temporais diferentes.

Entretanto, há uma questão que não está resolvida: a irreversibilidade dos processos biológicos. A teoria dos sistemas dinâmicos trata de situações em que o sentido da seta do tempo é irrelevante e, portanto, é incompatível com os processos biológicos reais. ${ }^{8}$

Talvez a solução para isso fosse uma combinação da teoria dos sistemas dinâmicos com a termodinâmica, por meio de um enunciado adequado de sua segunda lei (Leff \& Rex, 1990).

Outra opção seria lançar mão da teoria da informação de Shannon, construindo alguns conceitos do tipo entropia informacional, capazes de medir a influência do tempo na organização dos parâmetros do sistema biológico. Nesse ponto, a idéia de auto-organização, tão popular entre os biólogos, pode agrupar-se aos conceitos biomatemáticos que podem emergir. ${ }^{9}$

\section{Imprevisibilidade: visão clássica e quântica}

Apesar de todos os pontos positivos e todas as perspectivas de bom uso das teorias matemáticas discutidas anteriormente, resta ainda uma questão importante. Como modelar a imprevisibilidade nos processos biológicos, sua extrema sensibilidade às condições internas e do ambiente e, principalmente, a grande possibilidade de comportamentos que, embora parecidos, apresentam sempre uma variabilidade notável?

7 Ruele, 1991; Gleick, 1988; Bai-Lin, 1990; Stewart, 1989.

8 Gould, 1987; Coveney \& Highfield, 1990; Prigogine \& Stengers, 1984.

9 Prigogine \& Stengers, 1984, 1992; Shannon \& Weawer, 1949; Bohm \& Peat, 1987; Atlan, 1992. 
A teoria dos sistemas dinâmicos tenta dar uma resposta a essas questões por meio do conceito de caos determinístico, baseado na conjectura de Poincaré, já citada. Sistemas descritos por equações determinísticas apresentam, em razão de suas nãolinearidades, comportamentos sensíveis às condições iniciais.

Isto é, duas condições iniciais praticamente idênticas produzem, ao longo do tempo, respostas completamente diferentes, introduzindo, portanto, a imprevisibilidade e variabilidade desejadas nos modelos. A esse fenômeno tem sido dado o nome de caos determinístico. ${ }^{10}$

Essa é uma maneira de explicar a questão da imprevisibilidade usando os mesmos fundamentos da mecânica clássica, e lançando mão de equações determinísticas.

Há, entretanto, uma corrente de pesquisadores fortemente inclinados a tratar o problema de imprevisibilidade biológica por meio das idéias originárias da mecânica quântica, introduzindo formalismos estocásticos no trato dos problemas (Berg, 1991).

O ponto de encontro dessas duas linhas de raciocínio talvez seja a utilização da teoria ergódica, complementando a teoria de bifurcações com um tratamento probabilístico dos parâmetros e dos estados, via equações diferenciais estocásticas (Haken, 1980).

\section{Conclusões}

Esse trabalho tentou apresentar, de maneira qualitativa, e sem recorrer a equações, uma visão dos principais métodos da biomatemática, enfatizando a teoria dos sistemas dinâmicos não lineares, complementada com alguma ferramenta adicional, visando a contornar as dificuldades naturais impostas pela natureza dos processos biológicos.

Para a irreversibilidade, a segunda lei da termodinâmica; para a diversidade de escalas temporais, os conceitos de dinâmica rápida e lenta.

A complexidade, necessitando incorporar conceitos formais de organização biológica e de sensibilidade às condições iniciais e de contorno, pode fazê-lo por meio das idéias de caos determinístico e de abordagens quânticas.

\section{Agradecimentos}

Agradeço ao IEA-USP, ao Laboratório de Automação e Controle da EP-USP e ao Grupo Multidisciplinar de Ritmos Biológicos do ICB-USP, onde tenho encontrado respaldo para estudar biomatemática; e ao Grupo de Auto-Organização e Complexidade do CLE-HC da Unicamp, onde iniciei meu interesse pelo assunto.

10 Holden, 1993; Ruele, 1991; Gleick, 1988; Bai-Lin, 1990; Fiedler-Ferrara \& Prado, 1994; Ott, 1993; Stewart, 1989. 
PIQUEIRA, J. R. C. Biomathematics: methods and limits. Trans/Form/Ação (São Paulo), v.19, p.141-149, 1996.

- ABSTRACT: This article tries to give a general vision of the mathematical methods used to model biological processes. There are two kinds of methods: deterministics using dynamical systems theory and probabilistics, using stochastic differential equations. However, three points can not be neglected for biological processes: complexity, unpredictability and diversity of time scales.

- KEYWORDS: Dynamical systems; complexity; unpredictability; differential equations; classical mechanics; quantum mechanics; thermodynamic.

\section{Referências bibliográficas}

ATLAN, H. Entre o cristal e a fumaça. Rio de Janeiro: Zahar, 1992.

BAI-LIN, H. Chaos-II. Singapure: World Scientific Publishing Co., 1990.

BASSANEZI, R. C., FERREIRA JÚNIOR, W. C. Equações diferenciais. São Paulo: Harbra, 1988. BERG, H. C. Random Walks in Biology. New Jersey: Princeton University Press, 1991.

BOHM, D., PEAT, F. D. Science, Order and Creativity. New York: Bantam Books, 1987.

BLUMENFELD, L. A. Problems of Biological Physic. Berlin: Springer, 1980.

COVENEY, P., HIGHFIELD, R. The Arrow of Time. New York: Ballantine Books, 1990.

CVITANOVIC, P. Universality in Chaos. 2.ed. Bristol: Adam-Hilger, 1989.

ENGEL, A. B. Elementos de biomatemática. Washington, D.C.: Secretaria Geral da OEA, 1978 FIEDLER-FERRARA, N., PRADO, C. do. Caos: uma introdução. São Paulo: Edgar-Blucher, 1994.

GLEICK, J. Chaos. London: Heinemann, 1988.

GOULD, S. J. Time's Arrow Time's Cycle. Cambridge: Harward University Press, 1987.

HAKEN, H. Dynamics of Synergetic Systems. Berlin: Springer, 1980.

HIRSH, M. W., SMALE, S. Differential Equations, Dynamical Systems, and Linear algebra. Orlando: Academic Press, 1974.

HOBSON, J. A. The Dreaming Brain. New York: Harper-Collins Publishers, 1988.

HOLDEN, A. H. Chaos. New York: Princeton University Press, 1993.

KAUFFMAN, S. A. The Origins of Order. New York: Oxford University Press, 1993.

KURAMOTO, Y. Chemical Oscillations, Waves Turbulence. Berlin: Springer, 1980.

LEFF, H. S., REX, A. F. Maxwell's Demon. New Jersey: Princeton University Press, 1990.

MITTENTHAL, J. E., BASKIN, A. B. The Principles of Organization in Organisms. Santa Fé: Addison-Wesley Publishing Co., 1992.

MURRAY, J. D. Mathematical Biology. New York: Springer, 1993.

OTT, E. Chaos in Dynamical Systems. New York: University Press, 1993.

PRIGOGINE, I., STENGERS, I. Order out of Chaos. New York: Bantam Books, 1984. Entre o tempo e a eternidade. São Paulo: Cia. das Letras, 1992.

RUELE, D. Chance and Chaos. New Jersey: Princeton University Press, 1991.

SHANNON, C. E., WEAWER, W. The Mathematical Theory of Communication. Chicago: University of Illinois Press, 1949.

SOLOMON, M. E. Dinâmica de populações. São Paulo: EPU, 1981. 
STEWART, I. Does God Play Dice. Cambridge, Mass.: Basil Blackwell, 1989.

THOM, R. Structural Stability and Morphogenesis. Reading, Mass.: The Benjamin / Cummings Publishing Company, 1975.

THOMPSON, D. Sobre el crecimiento y la forma. Madrid: Blume Ediciones, 1980.

WEBER, B. H., DEPEW, D. J., SMITH, J. D. Entropy, Information and Evolution. Cambridge, Mass.: MIT Press, 1988.

WIGGINS, S. Introduction to Applied Nonlinear Dynamical Systems and Chaos. New York: Springer Verlag, 1990. 